06

\title{
Физические основы формирования гетероваризонной структуры на основе кремния
}

\author{
() М.К. Бахадирханов, С.Б. Исамов
}

Ташкентский государственный технический университет, 100095 Ташкент, Узбекистан

e-mail: bahazeb@yandex.ru

Поступило в Редакцию 15 марта 2021 г.

В окончательной редакции 23 апреля 2021 г.

Принято к публикации 28 мая 2021 г.

C формированием бинарных элементарных ячеек на основе элементов $\mathrm{A}^{\mathrm{II}}$ и $\mathrm{B}^{\mathrm{VI}}, \mathrm{A}^{\mathrm{III}}$ и $\mathrm{B}^{\mathrm{V}}$ в приповерхностной области кремния была получена гетероваризонная структура, не разрушающая кристаллическую структуру, без поверхностных состояний толщиной около $5 \mu \mathrm{m}$. Полученная гетероваризонная структура обладает особыми фундаментальными параметрами, обеспечивающими поглощение света в широком интервале солнечного спектра от УФ до ИК излучения с $\lambda=0.1-3 \mu \mathrm{m}$, т. е. охватывает весь спектр Солнца.

Ключевые слова: кремний, фотоэлемент, солнечный элемент, гетероваризонная структура, фотовольтаика, соединения $\mathrm{A}^{\mathrm{II}}$ и $\mathrm{B}^{\mathrm{VI}}, \mathrm{A}^{\mathrm{III}}$ и $\mathrm{B}^{\mathrm{V}}$.

DOI: 10.21883/JTF.2021.11.51528.60-21

\section{Введение}

Благодаря именно кремнию современная электроника достигла очень больших успехов. Все это связанно с достаточно большими запасами кремния на земном шаре, а самое главное освоена доступная технология получения кремневых кристаллов с необходимой чистотой и электрическими параметрами и заданными размерами монокристаллов. Ни для одного полупроводникового материалов ближайшее время не будет разработана технология получения кристаллов такой чистоты и с такими геометрически размерами.

Фотоэнергетика может стать основным источником энергии для человечества не только в далеком будущем, но и в ближайшее время, так как это самый чистый и вечный источник энергии. В настоящее время в фотоэнергетике кремний был и остается основным материалом, более $90 \%$ фотоэлектрических станций работают на основе кремниевых фотоэлементов. Однако широкомасштабное использование кремниевых фотоэлементов в фотоэнергетике в наземных условиях ограничено их низким КПД, которое достигло в промышленности 20\%, а в лабораторных условиях 25\% [1-3].

Фотоэлементы на основе полупроводниковых соединений $\mathrm{A}^{\mathrm{III}} \mathrm{B}^{\mathrm{V}}$ и $\mathrm{A}^{\mathrm{II}} \mathrm{B}^{\mathrm{VI}}$ хоть и имеют достаточно высокий КПД, особенно многокаскадные фотоэлементы [4-5], но они не могут быть использованы как источник энергии в наземных условиях, т.е. практически не возможно использовать такие фотоэлементы в более широком масштабе. Это связанно не только с очень высокой ценой таких фотоэлементов (они почти в 50 раз дороже, чем фотоэлементы на основе кремния), но и с ограниченным запасом элементов в составе полупроводников $\mathrm{A}^{\mathrm{III}} \mathrm{B}^{\mathrm{V}}$ и $\mathrm{A}^{\mathrm{II}} \mathrm{B}^{\mathrm{VI}}$, их токсичностью, а также слишком сложной технологией получения таких элементов [6-8].
Поэтому единственным выходом остается повышение КПД фотоэлементов на основе кремния или разработка нового класса материла на его основе для будущего фотоэнергетики в наземных условиях.

Более глубокий анализ показывает, что низкое значение КПД кремниевых солнечных элементов в основном связано с фундаментальными параметрами этого материала, т. е. небольшой шириной запрещенной зоны, не прямозонной структурой, не высоким значением подвижности по сравнению с полупроводниковыми соединениями $\mathrm{A}^{\mathrm{III}} \mathrm{B}^{\mathrm{V}}[9]$.

Поэтому можно однозначно утверждать, что без изменения фундаментальных параметров кремния практически невозможно создать более эффективные фотоэлементы [10,11].

Насколько нам известно, в настоящее время не существуют какие-либо физические материалы и технологии, позволяющие изменить фундаментальные параметры кремния, а также разработать какую-либо технологию получения новых перспективных материалов для будущего фотоэнергетики.

Целью настоящей работы являлось физическое обоснование возможности создания нового типа гетероваризонных структур за счет формирования бинарных кластеров атомов III и V групп в решетке кремния, а также раскрытие новых физических и функциональных возможностей такого материала для оптоэлектроники и фотоэнергетики.

\section{1. Физические основы формирования бинарных элементарных ячеек в решетке кремния}

В последние 10-15 лет ученые кафедры „Цифровая электроника и микроэлектроника“ ТГТУ занимаются 
возможностью управления фундаментальными параметрами кремния [12-14]. Ученым этой кафедры удалось физически обосновать оригинальную и доступную технологию получения кремния с управляемыми фундаментальными параметрами. Суть данного технологического решения заключается в формировании в решетке кремния бинарных нанокластеров, т. е. бинарных элементарных ячеек с управляемыми параметрами.

Как известно, элементы III и V групп в кремнии в основном находятся в узлах подрешетки и создают в запрещенной зоне кремния мелкие донорные и акцепторные энергетические уровни. Растворимость этих элементов в кремнии имеет достаточно высокое значение и достигает $N \geq 10^{21} \mathrm{~cm}^{-3}$. В атомарном состоянии они находятся в виде однократно заряженных ионов $\mathrm{A}^{\mathrm{III}-}, \mathrm{B}^{\mathrm{V}+}$ и вокруг себя создают электрический потенциал, также они создают соответствующую дополнительную концентрацию носителей заряда как в зоне проводимости, так и валентной зоне. Все это приводит к существенному нарушению термодинамически равновесного состояния кристалла. Поэтому кристалл в таких условиях находится в термодинамически неравновесном состоянии [15].

Установлено, что можно создать такие термодинамические условия диффузионного легирования кремния, последовательно элементами III и V групп, а также оптимальные термодинамические условия термоотжига, после диффузионного легирования, позволяющего находиться атомам III и V групп в решетке кремния рядом, т.е. они будут занимать места в соседних узлах решетки (рис. 1).

Как видно, при этом формируются электронейтральные молекулы между ионами $\mathrm{A}^{\mathrm{III}-}$ и $\mathrm{B}^{\mathrm{V}+}$. Формирование таких молекул полностью экранирует электрический потенциал каждого иона атомов III и V группы. При этом атомы III и V групп в кремнии действуют не как примесные атомы, а как основные атомы новой элементарной ячейки. Поэтому атомы III и V групп не создают энергетические уровни в запрещенной зоне, т. е. наличие их не создает дополнительные электроны и дырки как в зоне проводимости, так и валентной зоне. Формирование

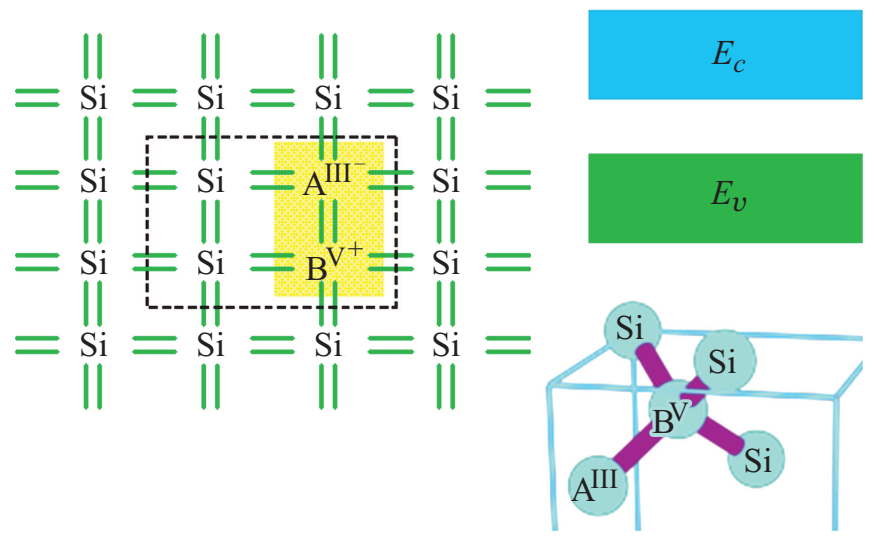

Рис. 1. Электронейтральные молекулы между ионами $\mathrm{A}^{\mathrm{III}-}$ и $\mathrm{B}^{\mathrm{V}+}$ и формирование новых элементарных ячеек $\mathrm{Si}_{2} \mathrm{~A}^{\mathrm{III}} \mathrm{B}^{\mathrm{V}}$ в кремнии. таких молекул не нарушает тетраэдрическую связь в решетке кремния. Это означает, что формирование таких электронейтральных молекул между ионами элементов III и V групп обеспечивает более выгодное термодинамическое равновесное состояние решетки. Это приводит к существенному стимулированию самоорганизации формирования таких электронейтральных молекул между ионами элементов $\mathrm{A}^{\mathrm{III}}$ и $\mathrm{B}^{\mathrm{V}}$.

Формирование таких электронейтральных молекул интересно тем, что при этом в решетке кремния появляется новая элементарная ячейка $\mathrm{Si}_{2} \mathrm{~A}^{\mathrm{III}-} \mathrm{B}^{\mathrm{V}+}$ (рис. 1). Эти элементарные ячейки в отличие от элементарной ячейки кремния, которая имеет $100 \%$ ковалентную связь, обладают частично ионно-ковалентной связью, т. е. появляется элементарная ячейка, не существующая в природе. Ионная доля в таких новых бинарных элементарных ячейках $\mathrm{Si}_{2} \mathrm{~A}^{\mathrm{III}-} \mathrm{B}^{\mathrm{V}+}$, согласно $[15,16]$, определяется разностью значений электроотрицательности атомов III и V групп. Расчеты показывают, что при значении разности электроотрицательности в пределах $\Delta x \leq 1$ доля ионной связи в них составляет около $15-20 \%$. Это означает, что при этом ковалентная связь в них будет преобладающей.

Теперь возникает вопрос все ли атомы элементов III и V групп могут образовывать электронейтральные молекулы и будут ли формировать новые бинарные элементарные ячейки в решетке кремния? Теоретически да, но при этом для этого необходимо чтобы выполнялись следующие условия $[17,18]$.

1. Сумма ковалентных радиусов атомов элементов III $\left(r_{\mathrm{III}}\right)$ и $\mathrm{V}\left(r_{\mathrm{V}}\right)$ групп должна быть достаточно близка к сумме ковалентных радиусов двух атомов кремния $\left(r_{\mathrm{Si}}\right)$, т. е. должны выполняться следующие соотношения: $r_{\mathrm{III}}+r_{\mathrm{V}}-2 r_{\mathrm{Si}} \leq \pm 0.2 r_{\mathrm{Si}}, 2 r_{\mathrm{Si}}-\left(r_{\mathrm{III}}+r_{\mathrm{V}}\right) \leq \pm 0.2\left(2 r_{\mathrm{Si}}\right)$.

2. Разность значений электроотрицательности атомов элементов III и V групп не должна существенно отличаться от значений электроотрицательности атомов в кремнии.

3. Растворимость атомов элементов III и V групп и их коэффициенты диффузии не должны сильно отличаться друг от друга.

4. Необходимо проведение термоотжига после диффузионного легирования материала. Термодинамические условия термоотжига (температура и время) в основном определяются химическими и физическими параметрами элементов III и V групп.

На основе анализа электрических и химических параметров элементов III и V групп, а также их коэффициентов диффузии и растворимости в кремнии установлены следующие группы пар элементов III и V групп. Эти данные показывают возможность формирования достаточно широкого класса бинарных элементарных ячеек с участием атомов III и V групп.

1. Наиболее подходящие пары - BBi, AlP, GaP, BP.

2. Подходящие пары - BSb, AlAs, GaAs.

3. Возможные пары - BAs, GaSb, InP.

4. Не подходящие пары - AlSb, InAs, InSb. 


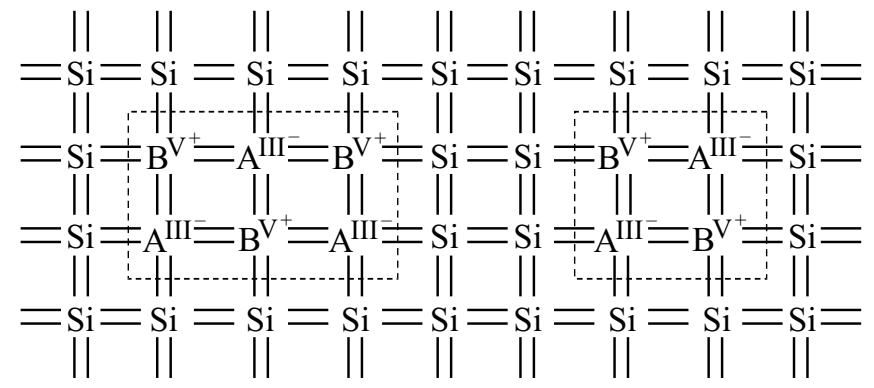

Рис. 2. Некоторые возможные комбинации бинарных элементарных ячеек в решетке кремния.

Как известно, фундаментальные параметры каждого полупроводникового материала определяются его структурой, составом и химической связью элементарных ячеек, из которых состоит полупроводниковый материал. Поэтому необходимо выбрать оптимальные условия диффузионной технологии легирования кремния атомами элементов III и V групп, позволяющую вводить с максимальной и одинаковой концентрацией, а также распределять эти атомы в образцах кремния с учетом их коэффициентов диффузии как в процессе одновременной, так и последовательной диффузии элементов III и V групп. T. е. необходимо определить оптимальные температуры и время дополнительного термоотжига, обеспечивающее максимальное участие введенных примесных атомов в формировании электронейтральных молекул.

С повышением концентраций введенных атомов элементов III и V групп существенно увеличивается вероятность формирования электронейтральных атомов между атомами элементов III и V групп, следовательно, увеличивается концентрация бинарных элементарных ячеек, а это в свою очередь приводит к появлению некоторых возможных комбинаций бинарных элементарных ячеек (рис. 2).

Энергетические связи атомов в элементарных ячейках и их комбинации будут существенно отличаться друг от друга. Это означает, что необходимая энергия для освобождения электронов в таких элементарных ячейках с различными их комбинациями существенно отличается, т.е. будут существенно различные значения ширины запрещенной зоны. Поэтому в них не будет одинакового фиксированного значения длины волны света для фундаментального поглощения $\left(\lambda_{b}\right)$, каждая из них будет иметь свои значения $\lambda_{b}$, т.е. существенно расширяется спектр фундаментального поглощения кремния. Спектр поглощения будет заметнее только при достаточно высокой концентрации таких элементарных ячеек и их комбинаций.

При введении максимальной концентрации примесных атомов III и V групп $N \geq 10^{21} \mathrm{~cm}^{-3}$ в кремний, обеспечивающее их одинаковое концентрационное распределение, в отличие от выше указанных различных комбинаций бинарных элементарных ячеек могут формироваться нанокристаллы полупроводникового соединения на основе введенных примесных атомов элементов III и V групп (рис. 3). Это означает, что появляется локальная область, обогащенная нанокристаллами атомов полупроводниковых соединений $\mathrm{A}^{\mathrm{III}} \mathrm{B}^{\mathrm{V}}$, погруженная в решетку кремния. Размер нанокристаллов полупроводниковых соединений $\mathrm{A}^{\mathrm{III}} \mathrm{B}^{\mathrm{V}}$ может изменяться от нескольких нанометров до нескольких десятков нанометров в зависимости от условий легирования. Энергетическая структура кремния с высокой концентрацией бинарных элементарных ячеек и таких структур существенно меняется, и кремний превращается в новый материал.

Наиболее вероятные структуры на основе распределения элементарных ячеек по глубине кремния $\mathrm{Si}_{2} \mathrm{~A}^{\mathrm{III}-} \mathrm{B}^{\mathrm{V}+}$ представлены на рис. 4. Как видно в приповерхностной области толщиной $d=1-100 \mathrm{~nm}$, появляется область, обогащенная нанокристаллами с различным размером. Дальше от 0.1 до $1 \mu \mathrm{m}$ появляется область, обогащенная различными комбинациями бинарных элементарных ячеек. После этой области существует область, обогащенная в основном отдельными элементарными ячейками $d=1-1.5 \mu \mathrm{m}$, затем

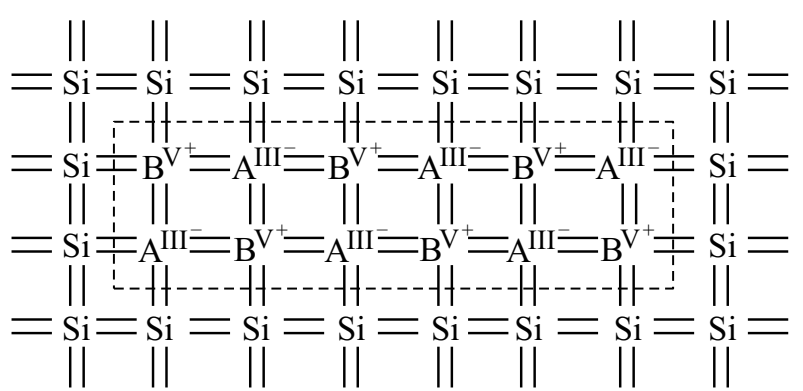

Рис. 3. Нанокристаллы полупроводникового соединения на основе элементов III и $\mathrm{V}$ групп в решетке кремния $\left(\mathrm{Si}_{2} \mathrm{~A}^{\mathrm{III}-} \mathrm{B}^{\mathrm{V}+}\right)$.

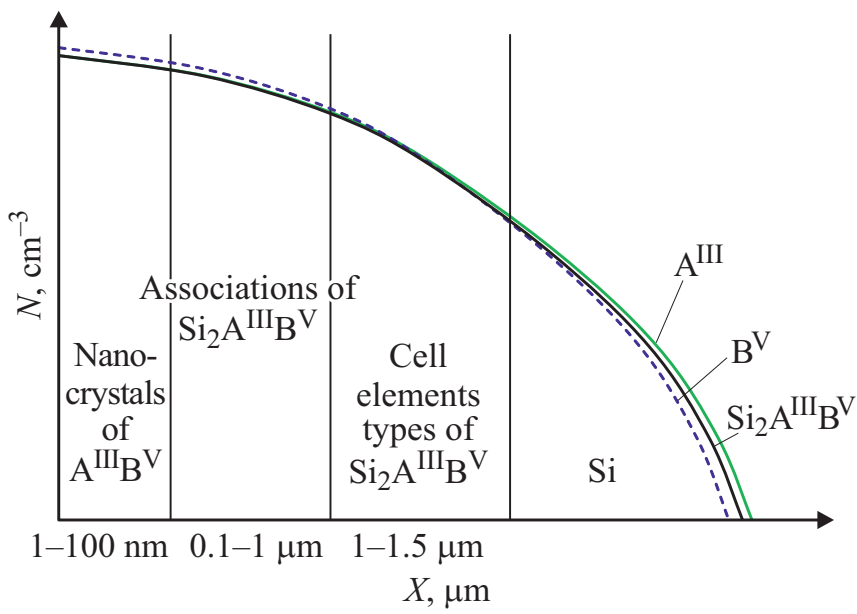

Рис. 4. Наиболее вероятные структуры на основе распределения элементарных ячеек по глубине кремния. $\mathrm{A}^{\mathrm{III}}$ концентрационное распределение элементов III группы после диффузии элементов V группы (первая стадия диффузии), $\mathrm{B}^{\mathrm{V}}$ - концентрационное распределение элементов V группы (вторая стадия диффузии), $\mathrm{Si}_{2} \mathrm{~A}^{\mathrm{III}} \mathrm{B}^{\mathrm{V}}-$ концентрационное распределение бинарных элементарных ячеек в решетке кремния. 

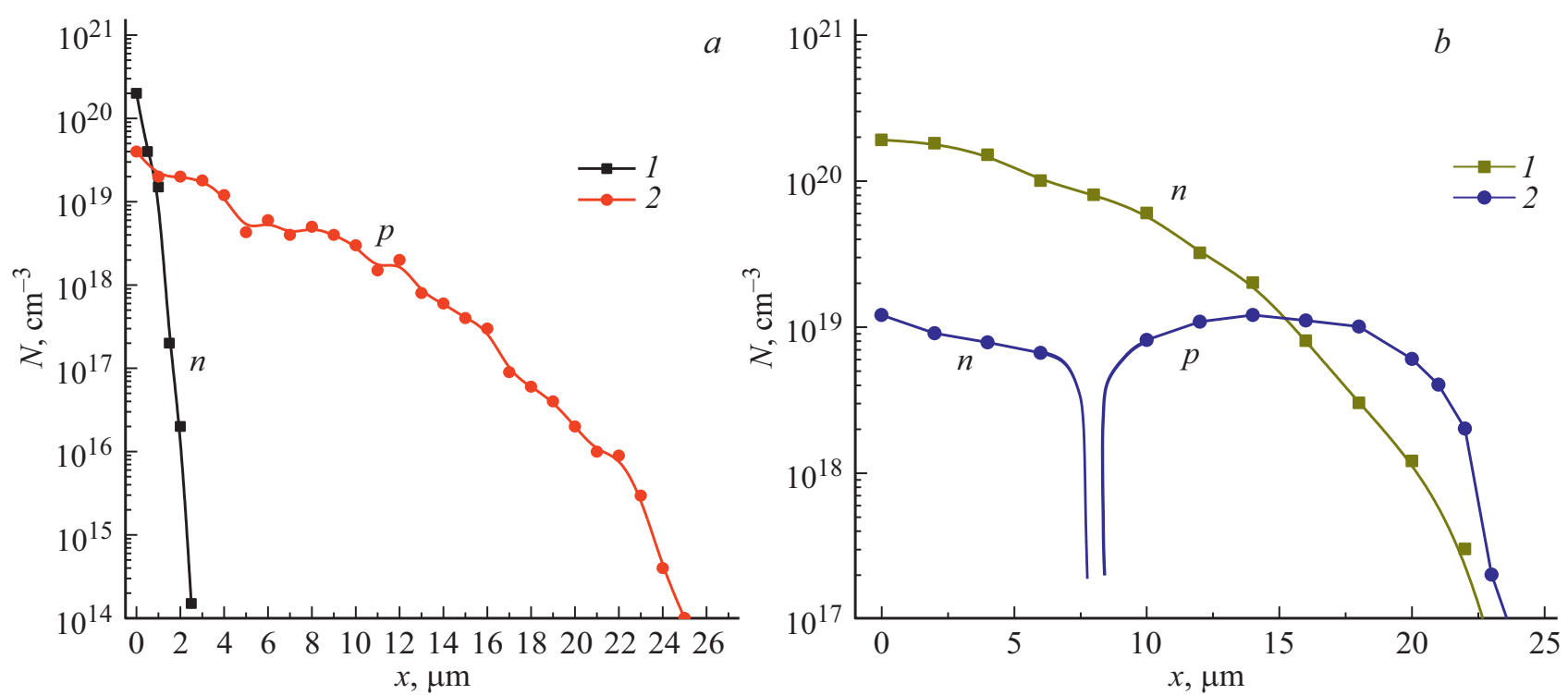

Рис. 5. Концентрационное распределение электронов (атомов фосфора) и дырок (галлия) в решетке кремния: $a-$ диффундированных независимо друг от друга, $b-$ при последовательной диффузии. Кривая 1 - распределение электронов (фосфора). Кривая 2 - распределение дырок (галлия).

до $d=1.5-3 \mu$ m следует область кремния с наименьшей концентрацией бинарных элементарных ячеек.

Толщиной каждой области и их распределением можно управлять в широком интервале в зависимости от условий диффузии. Таким образом, из рис. 5 видно, что в кремнии, начиная от поверхности до глубины $X$, появляется область, в которой структура, состав и химические связи меняются от полупроводниковых соединений $\mathrm{A}^{\mathrm{III}} \mathrm{B}^{\mathrm{V}}$ до чистого кремния без существенного изменения кристаллической структуры кремния и тетраэдрической связи, но естественно свойства каждой структуры различаются, и они существенно отличаются фундаментальными параметрами друг от друга.

Эта структура не похожа на существующие варизонные структуры и не похожа на гетероструктуру, т. е. возникает структура нового типа - гетероваризонная структура без всяких нарушений структуры и поверхностных состояний. Это структура создается с помощью диффузионной технологии, так как здесь применяется диффузионный способ формирования структур.

Для доказательства формирования таких гетероваризонных структур были проведены предварительные эксперименты с участием примесных атомов галлия и фосфора, входящих в ряд более подходящих пар. В качестве исходного материала был использован монокристаллический кремний КЭФ-100 $\left(N_{\mathrm{P}} \sim 10^{13} \mathrm{~cm}^{-3}\right)$ с содержанием кислорода $N_{\mathrm{O}_{2}} \approx(5-6) \cdot 10^{17} \mathrm{~cm}^{-3}$ и плотностью дислокаций $N_{D} \sim 10^{3} \mathrm{~cm}^{-2}$. Размер образцов составлял $V \sim 1 \times 4 \times 8 \mathrm{~mm}$. После необходимых механических и химических обработок образцов проводилась диффузия фосфора из нанесенного слоя фосфорнокислого аммония на воздухе при $T=1000^{\circ} \mathrm{C}$ в течение $t=2 \mathrm{~h}$. После диффузии во всех образцах снималось с поверхности фосфоросиликатное стекло методом трав- ления с $\mathrm{HF}$ и $\mathrm{NH}_{4} \mathrm{~F}$. Концентрации атомов фосфора и галлия исследовались 4-х зондовым методом. При этом предполагалось, что эти атомы находятся в решетке в электроактивном состоянии. Наряду с этим учитывались зависимости подвижности носителей заряда от концентрации примесных атомов. При этом концентрация электронов (фосфора) на поверхности образцов составляла $N_{\mathrm{P}}=2 \cdot 10^{20} \mathrm{~cm}^{-3}$.

Диффузия галлия в кремнии (КЭФ 100) проводилась из газовой фазы при температуре $T=1250^{\circ} \mathrm{C}$ в течение $4 \mathrm{~h}$. Такой выбор диффузии галлия дает возможность получить максимальную концентрацию на поверхности и в объеме кремния.

Затем в образцах, легированным фосфором, проводилась диффузия галлия при $T=1250^{\circ} \mathrm{C}$ в течение $4 \mathrm{~h}$. При этом одновременно подвергались термоотжигу образцы кремния, легированного фосфором (без галлия), также при $T=1250^{\circ} \mathrm{C}, t=4 \mathrm{~h}$, чтобы определить изменения концентрационного распределения фосфора при дополнительных отжигах.

На рис. 5, a представлено концентрационное распределение атомов фосфора в кремнии после диффузии $T=1000^{\circ} \mathrm{C}, t=2 \mathrm{~h}$. Также представлено концентрационное распределение галлия после диффузии $T=1250^{\circ} \mathrm{C}, t=4 \mathrm{~h}$ в образцах КЭФ-100.

Как видно из экспериментальных результатов, концентрация фосфора в приповерхностной области составляет $N_{\mathrm{P}}=2 \cdot 10^{20} \mathrm{~cm}^{-3}$, при этом на глубине $x=2.5 \mu \mathrm{m}$, его концентрация уменьшена до $\sim 10^{14} \mathrm{~cm}^{-3}$ (кривая 1) при этом образцы все время остаются $n$-типом.

Полученные данные соответствуют литературным данным [12-14]. Как видно из рисунка, концентрационное распределение фосфора и галлия при их диффузии по отдельности существенно отличаются друг от друга. 
На рис. 5, $b$ представлено концентрационное распределение электронов (фосфора) в контрольных образцах, которые подвергались дополнительному термоотжигу при $T=1250^{\circ} \mathrm{C}, t=4 \mathrm{~h}$ (кривая 1 ), а также концентрационное распределение носителей заряда в образцах кремния, легированного галлием, при $T=1250^{\circ} \mathrm{C}$, $t=4 \mathrm{~h}$, которые были предварительно легированы фосфором при $1000^{\circ} \mathrm{C}, t=2 \mathrm{~h}$ (кривая 2). Как видно в результате дополнительного отжига, при $T=1250^{\circ} \mathrm{C}$, $t=4 \mathrm{~h}$ поверхностная концентрация фосфора незначительно уменьшается, а глубина проникновения достигает $x=25 \mu \mathrm{m}$ (кривая 1 ), в области $x=0-25 \mu \mathrm{m}$ образцы приобретают явно $n$-тип.

Концентрационное распределение в образцах легированных галлием при $T=1250^{\circ} \mathrm{C}, t=4 \mathrm{~h}$, после легирования фосфором при $1000^{\circ} \mathrm{C}, t=2 \mathrm{~h}$ (рис. $5, b$, кривая 2) показывает, что в исследуемых образцах до глубины $x=7.5-8 \mu \mathrm{m}$ имеется $n$-тип проводимости. При этом концентрация электронов (фосфора) существенно уменьшается, а при $x>7.5-8 \mu \mathrm{m}$ образцы приобретают $p$-тип проводимости. В области $x=7.5-10 \mu \mathrm{m}$, концентрация дырок незначительно увеличивается, а в области $x=8-15 \mu \mathrm{m}$ концентрация дырок (галлия) практически остается постоянной, а при $x>17 \mu \mathrm{m}$ достаточно резко уменьшается.

Эти полученные результаты показывают, что при диффузии галлия (при наличии высокой концентрации фосфора), концентрация фосфора в области $x=0-7.5 \mu \mathrm{m}$ уменьшается на 1.5-1 порядок, затем концентрация галлия становится больше чем фосфора и материал обладает р-типом проводимостью. Хотя как видно из рис. 5, $b$ (кривая 1 ) концентрация фосфора до области $15 \mu \mathrm{m}$ больше, чем концентрация галлия. Эти результаты дают возможность предполагать, что наличие фосфора в кремнии приводит к повышению концентрации галлия

\section{2. Обсуждение}

Полученные экспериментальные результаты невозможно объяснить взаимной компенсацией донорных (фосфор) и акцепторных (галлий) примесных атомов, так как атомы фосфора и галлия в решетке кремния распределены хаотически и пространственно разделены, что соответственно не должно приводить к повышению концентрации атомов галлия при наличии атомов фосфора. Поэтому можно предлагать, что эти явления связаны с взаимодействием атомов фосфора и галлия. Поскольку атомы фосфора в решетке кремния находятся в узлах кристаллической решетки в виде положительно заряженных ионов $\mathrm{P}^{+}$, создавая дополнительные электроны в зоне проводимости, концентрация которых равна $N_{\mathrm{P}^{+}}=n$. Наличие достаточно большой концентрации положительно заряженных атомов фосфоpa $\left(\mathrm{P}^{+}\right)$создает значительный электрический потенциал. Этот потенциал распределен от поверхности кристалла вглубь образца кремния, что стимулирует повышение

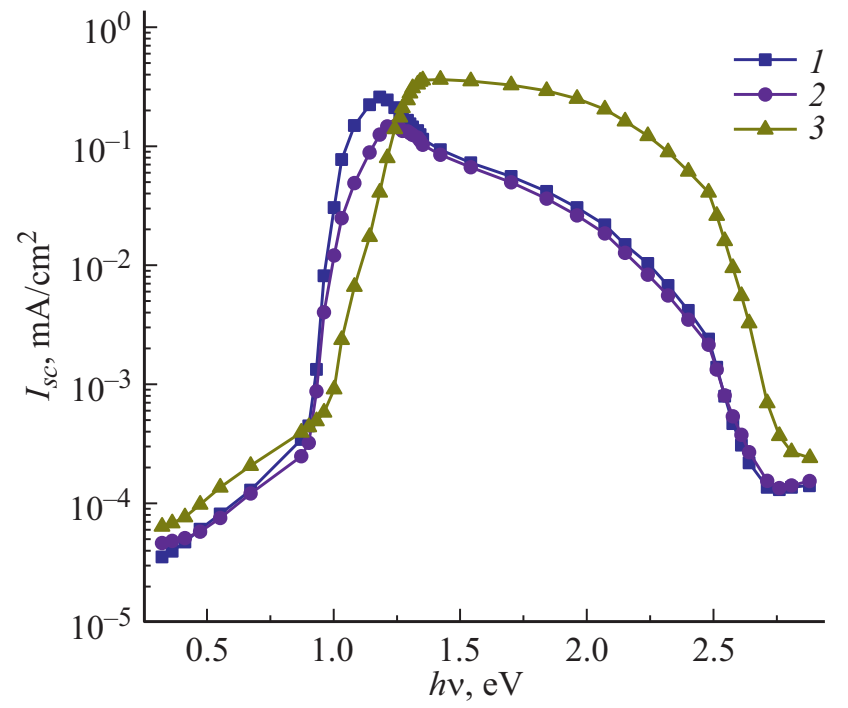

Рис. 6. Спектральная зависимость тока короткого замыкания образцов кремния с $p-n$-переходом, полученным с помощью: $1-\mathrm{P}, 2-\mathrm{Ga}$ и $3-\mathrm{GaP}$.

концентрации атомов галлия в процессе диффузии, которые в кремнии действуют как акцепторная примесь в виде отрицательных ионов $\mathrm{Ga}^{-}[19,20]$. Поэтому можно предполагать, что в результате таких взаимодействий в решетке кремния появляются донорно-акцепторные комплексы, т. е. квазимолекулы в виде $\left[\mathrm{P}^{+} \mathrm{Ga}^{-}\right]$. В свою очередь, квазимолекулы $\left[\mathrm{P}^{+} \mathrm{Ga}^{-}\right]$в решетке кремния и их распределение по глубине образуют гетероваризонную структуру, которые изменяют энергетическую структуру кремния.

Для оценки вклада в энергетические параметры кремния с гетероваризонными структурами на основе $\left[\mathrm{P}^{+} \mathrm{Ga}^{-}\right]$мы создали $p-n$-переходы в кремнии с участием примесных атомов галлия и фосфора по отдельности, и с гетероваризонными структурами. При этом в качестве исходного материала был использован монокристаллический кремний дырочного типа проводимости легированный бором марки КДБ-0.5 (для легирования фосфором) и монокристаллический кремний электронного типа проводимости легированный фосфором марки КЭФ-0.3 (для легирования галлием), гетероваризонная структура создавалась на основе кремния КДБ-0.5. Диффузия во всех образцах была проведена при одинаковых условиях $T=1250^{\circ} \mathrm{C}, t=0.5 \mathrm{~h}$.

Результаты эксперимента (рис. 6) показывают, что в $p-n$-переходах, образованных галлием и фосфором, их спектральные зависимости тока короткого замыкания $\left(I_{s c}\right)$ соответствуют друг другу. В случае сформированной квазимолекулы $\mathrm{Ga}^{-} \mathrm{P}^{+}$в кремнии $I_{s c}(h v)$ существенно отличается от легированного фосфором и галлием в отдельности.

1) Максимальное значение Isc в образцах с квазимолекулами $\mathrm{Ga}^{-} \mathrm{P}^{+}$наблюдается не при $h v=1.1 \mathrm{eV}$, а при $h v=1.35 \mathrm{eV}$; 
2) при этом наблюдаются существенно повышенные значения $I_{s c}$ в области $h v=1.3-2.8 \mathrm{eV}$;

3) имеет место расширение спектральной области чувствительности $I_{s c}$.

Полученные результаты позволяют предполагать, что такие существенные отличия $I_{s c}(h v)$ связаны с формированием бинарных элементарных ячеек $\mathrm{Si}_{2} \mathrm{GaP}$, в результате которых создается гетероваризонная структура на основе кремния. Так как формирование квазимолекулы $[\mathrm{GaP}]$ в решетке кремния не создает никакие энергетические уровни в запрещенной зоне кремния. Это также может служить доказательством формирования гетероваризонных структур с учетом галлия и фосфора в кремнии. Более достоверные данные могут дать результаты рентгеноструктурных и масс-спектроскопических анализов, над чем работают в настоящее время авторы.

Рассмотрим энергетическую структуру полученного материала в зависимости от природы введенных примесных атомов элементов III и V групп, где формируются нанокристаллы полупроводниковых соединений $\mathrm{A}^{\mathrm{III}} \mathrm{B}^{\mathrm{V}}$ с большими значениями энергии ширины запрещенный зоны как у GaP, BP, GaAs, а также с малыми значениями как у $\mathrm{GaSb}, \mathrm{InSb}$ и др. Это позволяет создать различные комбинации бинарных элементарных ячеек, т.е. получить структуру на глубине $d=0.1-5 \mu \mathrm{m}$ в кремнии с энергетической структурой, отличной от энергетической структуры полупроводниковых соединений $\mathrm{A}^{\mathrm{III}} \mathrm{B}^{\mathrm{V}}$. A также с изменяющейся шириной запрещенной зоны начиная от $\mathrm{GaP}, \mathrm{GaAs}, \mathrm{PB}$ до ширины запрещенной зоны кремния $E_{g}=1.12 \mathrm{eV}$, что обеспечивает максимальное поглощение УФ, видимой и ИК области светового излучения. Также формирование таких структур в приповерхностной области материала может образовывать соединения с запрещенной зоной меньше чем запрещенная зона кремния (GaSb, InAs, $\mathrm{InSb})$, в которой значение $E_{g}$ от поверхности кристалла непрерывно увеличивается до $E_{g}$ кремния на глубине $d$. В этом случае создается материал поглощающий ИК излучение с $\lambda>1.2 \mu \mathrm{m}$. При этом необходимо отметить, что ширина запрещенной зоны нанокристаллов $\mathrm{A}^{\mathrm{III}} \mathrm{B}^{\mathrm{V}}$ и различных комбинаций бинарных элементарных ячеек может отличаться от ширины запрещенной зоны как самих соединений $\mathrm{A}^{\mathrm{III}} \mathrm{B}^{\mathrm{V}}$, так и нанокристаллов $\mathrm{A}^{\mathrm{III}} \mathrm{B}^{\mathrm{V}}$, находящихся в кристаллической решетке кремния.

Таким образом, оптимальные условия легирования кремния различными парами атомов элементов III и V групп позволяют создать практически новый класс материалов с управляемой шириной запрещенной зоны на основе главного материала кремния.

Образование локальной области в решетке кремния, обогащенной нанокластерами $\mathrm{A}^{\mathrm{III}} \mathrm{B}^{\mathrm{V}}$, приводит к появлению в решетке кремния области с прямозонной структурой, похожей на зонную структуру соответствующих полупроводниковых соединений $\mathrm{A}^{\mathrm{III}} \mathrm{B}^{\mathrm{V}}$. Это означает, что существенно повышается коэффициент поглощения во всей области спектра Солнца. Таким образом, с формированием бинарных элементарных ячеек можно изменить более важный фундаментальный параметр кремния - зонную структуру, которую практически невозможно изменить какими либо другими методами.

В результате формирования бинарных элементарных ячеек с управляемыми параметрами в приповерхностной области можно получить достаточно толсты $(5 \mu \mathrm{m})$ слой кремния. С помощью материала с новыми фундаментальными параметрами, обеспечивающими поглощение света в широком интервале солнечного спектра от УФ до ИК излучения $\lambda=0.1-3 \mu \mathrm{m}$, охватывается весь спектр Солнца. Этим свойством не обладает ни один из существующих полупроводниковых материалов. Самое главное, что это получается на основе основного материала кремния.

\section{Заключение}

Особо следует отметить, что эта диффузионная технология получения таких материалов достаточно отработана, надежна, доступна, а самое главное она основана на действующей диффузионной планарной технологии, не требующей дорогостоящего оборудования, приборов и устройств.

Теперь несколько слов о функциональных возможностях кремния с бинарными, элементарными ячейками. Как показали предварительные расчеты, получение такого материала на основе кремния с бинарными элементарными ячейками с участием $\mathrm{Ga}-\mathrm{P}, \mathrm{P}-\mathrm{Al}$ и $\mathrm{B}-\mathrm{P}$ может увеличивать КПД фотоэлементов на 25-30\%. Расчеты также показывают, что при использовании оптимальной технологии, позволяющей получать элементарные ячейки с участием атомов GaP, GaAs, BP c необходимой концентрацией и распределением, можно изготовить фотоэлементы на основе кремния с КПД более $50 \%$. Это означает, что в ближайшем будущем (3-5 лет) дорогостоящие и сложные многокаскадные фотоэлементы на основе $\mathrm{A}^{\mathrm{III}} \mathrm{B}^{\mathrm{V}}$ будут заменены фотоэлементами с более высоким КПД на основе кремния с бинарными элементарными ячейками. Таким образом, предлагаемая научно-обоснованная модель управления фундаментальными параметрами кремния обеспечивает широкомасштабное использование фотоэнергетики в наземных условиях. Такие материалы также позволяют создать новые, надежные и дешевые приборы для оптоэлектроники.

\section{Конфликт интересов}

Авторы заявляют, что у них нет конфликта интересов.

\section{Список литературы}

[1] X. Ru, M. Qu, J. Wang, T. Ruan, M. Yang, F. Peng, W. Long, K. Zheng, H. Yan, X. Xu. Solar Energy Mater. Solar Cells, 215, 110643 (2020).

[2] M.A. Green, E.D. Dunlop, D.H. Levi, J. Hohl-Ebinger, M. Yoshita, A.W.Y. Ho-Baillie. Progr. Photovoltaics: Res. Applicat., 27, 565 (2019). 
[3] V.A. Milichko, A.S. Shalin, I.S. Mukhin, A.E. Kovrov, A.A. Krasilin, A.V. Vinogradov, P.A. Belov, C.R. Simovski. Phys. Usp., 59 (8), 727 (2016).

[4] Ж.И. Алфёров, Избранные труды. Нанотехнологии (Магистр-пресс, М., 2013)

[5] A. Louwen, W. Sark, R.Schropp, A. Faaij. Solar Energy Mater. Solar Cells, 147, 295 (2016).

[6] M. Yamaguchi, K.H. Lee1, K. Araki, N. Kojima. J. Phys. D: Appl. Phys., 51, 133002 (2018).

[7] S. Abdul Hadi, E.A. Fitzgerald, S. Griffiths, A. Nayfeh. J. Renewable Sustainable Energy, 10, 015905 (2018).

[8] М.А. Путято, Н.А. Валишева, М.О. Петрушков, В.В. Преображенский, И.Б. Чистохин, Б.Р. Семягин, Е.А. Емельянов, А.В. Васев, А.Ф. Скачков, Г.И. Юрко, И.И. Нестеренко. ЖТФ, 89 (7), 1071 (2019).

DOI: 10.21883/JTF.2019.07.47802.438-18

[9] K. Chen, R. Kapadia, A. Harker, S. Desai, J.S. Kang, S. Chuang, M. Tosun, C.M. Sutter-Fella, M. Tsang, Y. Zeng, D. Kiriya, J. Hazra, S.R. Madhvapathy, M. Hettick, Yu-Ze Chen, J. Mastandrea, M. Amani, S. Cabrini, Yu-Lun Chueh, J.W. Ager III, D.C. Chrzan, A. Javey. Nature Commun., 7, 10502 (2016). DOI: 10.1038/ncomms 10502

[10] Н.Д. Гудков. ЖТФ, 63 (5), 105 (1993).

[11] C.-X. Zhao, Y. Huang, J.-Q. Wang, C.-Y. Niu, Y. Jia. Phys. Lett. A. 383, 125903 (2019).

[12] M.K. Bakhadyrhanov, U.X. Sodikov, D. Melibayev, Tuerdi Wumaier, S.V.Koveshnikov, K.A. Khodjanepesov, Jiangxiang Zhan. J. Mater. Sci. Chem. Eng., 6, 180 (2018). DOI: $10.4236 / \mathrm{msce} .2018 .64017$

[13] M.K. Bakhadyrkhanov, S.B. Isamov, Kh.M. Iliev, S.A. Tachilin, K.U. Kamalov. Appl. Sol. Energy, 50 (2), 61 (2014).

[14] M.K. Bakhadyrhanov, U.X. Sodikov, Kh.M. Iliev, S.A. Tachilin, T. Wumaier, Mater. Phys. Chem., 1, 89 (2019).

[15] S. Adachi. Properties of group-IV, III-V and II-VI semiconductors (England: John Wiley \& Sons Ltd, 2005)

[16] Б.И. Болтакс. Диффузия и точечные дефекты в полупроводниках (Наука, Л., 1972)

[17] D.V. Saparov, M.S. Saidov, A.S. Saidov. Appl. Solar Energy. 52 (3), 236 (2016).

[18] A.S. Saidov, M.S. Saidov, Sh.N. Usmonov, K.T. Kholikov, D. Saparov. Appl. Solar Energy, 43 (3), 183 (2007).

[19] S. Haridoss, F. Bénière, M. Gauneau, A. Rupert. J. Appl. Phys., 51 (11), 5833 (1980).

[20] Y. Sato, I. Sakaguchi, H. Haneda. Jpn. J. Appl. Phys., 43 (12), 8024 (2004). 